\title{
Theoretical and Experimental Investigation of the Ramp Losses in Conductor and Coil Casing of the ATLAS Barrel Toroid Coils
}

\author{
J. J. Rabbers, A. Dudarev, R. Pengo, C. Berriaud, and H. H. J. ten Kate
}

\begin{abstract}
Each of the eight huge coils of the Barrel Toroid of the ATLAS detector consists of two double pancakes which are embedded in an aluminum alloy coil casing. The $57 \mathrm{~mm} \times 12 \mathrm{~mm}$ sized conductor is a Rutherford cable with NbTi-Cu strands co-extruded with a high purity aluminum stabilizer. The race track coils have overall dimensions of $25 \mathrm{~m} \times 5 \mathrm{~m}$ and the length of the conductor in the windings is $6.7 \mathrm{~km}$. The coils are conduction cooled with forced flow helium. The nominal operating current is $20.5 \mathrm{kA}$ and the nominal ramp rate is $4 \mathrm{~A} / \mathrm{s}$. During the test program of the individual coils the ramp losses are measured to confirm that they do not exceed the design cooling capacity of the ATLAS cryogenic system. The losses are determined from the amount of evaporated helium in the return flow. The ramp losses in the conductor consist of the hysteresis and coupling current losses in the Rutherford cable and eddy current loss in the pure aluminum stabilizer. Ohmic losses are generated in the coil casing which acts as a low resistive secondary of a transformer formed by the coil and the casing. In this paper the results of the loss measurements on the different coils, with different RRR (residual resistance ratio), are presented. Measurements are performed at various ramp rates. The results are in good agreement with the calculated losses, which are dominated by the loss in the coil casing.
\end{abstract}

Index Terms-AC loss, detector magnets, superconducting magnets.

\section{INTRODUCTION}

$\mathbf{T}$ HE eight race track coils of the ATLAS (A Toroidal LHC ApparatuS) Barrel Toroid were individually tested on-surface before assembling the Barrel Toroid (BT) in the underground cavern at CERN. The Barrel Toroid is already described many times in detail, see e.g. [1]. For clarity, here only a crosssection of the cold mass in the vacuum vessel is shown, see Fig. 1. The relevant coil parameters are listed in Table I. An overview of the test results can be found in [2], [3].

In this paper a study of the thermal loss during ramping of the current is presented. It is important for the operation of the Barrel Toroid that the heat load during ramp up and ramp down does not exceed the cooling capacity of the cryogenic system, which was designed on the basis of the estimated losses. A theoretical evaluation of the ramp losses in the conductor and the casing is presented. The experimentally obtained results for the different coils are compared with the theoretical predictions.

Manuscript received September 19, 2005.

J. J. Rabbers, A. Dudarev, R. Pengo, and H. H. J. ten Kate are with CERN, CH-1211 Geneva 23, Switzerland (e-mail: rabbers@cern.ch).

C. Berriaud is with CEA-Saclay, 91191, France.

Digital Object Identifier 10.1109/TASC.2005.864342

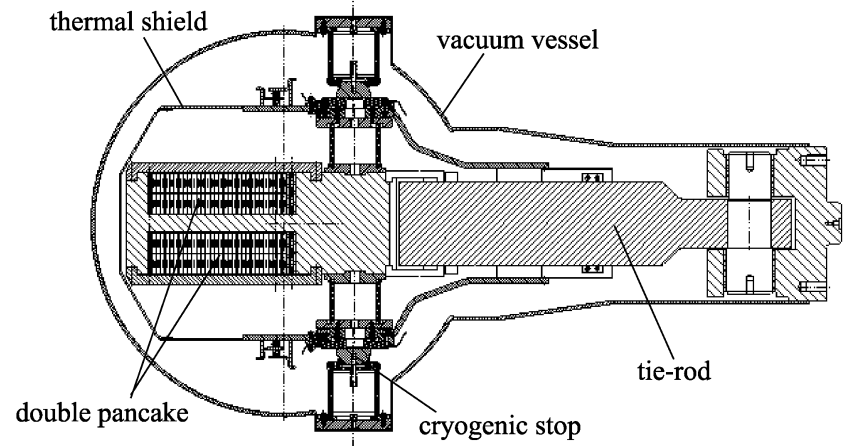

Fig. 1. Cross-section of double pancakes in coil casing, thermal shield, and vacuum vessel.

TABLE I

MAIN PARAMETERS OF BARREL TOROID COILS

Coil type

Total number of turns

Racetrack dimensions

Operating temperature

Nominal current

Self-inductance of single coil

Mutual ind.between coil and casing

Resistance of the casing @ $4.6 \mathrm{~K}$

Critical current margin $I / I_{C}$ at $4 \mathrm{~T}$

$2 \times$ double pancake
120
$25 \mathrm{~m} \times 5 \mathrm{~m}$
$4.6 \mathrm{~K}$
$20.5 \mathrm{kA}$
$0.56 \mathrm{H}$
$4.6 \mathrm{H}$ (including mirror)
$14 \mu \Omega$
$25 \%$

\section{LOSS FORMULAS}

During ramp up and ramp down of the magnet, losses are generated in the conductor and in the coil casing. The loss in the coil conductor consists of different types of loss: eddy current loss in the high purity aluminum stabilizer, interstrand coupling current loss in the Rutherford cable and hysteresis loss and interfilament coupling current loss in the strands. In the coil casing ohmic loss generated by the current that is induced in the casing.

In this section the loss formulas for each relevant loss component are given. A cross-section of the conductor with the definition of the dimensions is shown in Fig. 2 and the relevant parameters of the conductor are listed in Table II.

An experimental study of the conductor loss is published in [4], but for ramp rates which are orders of magnitude larger than the magnet ramp rate.

\section{A. Aluminum Stabilizer}

When the dimension of the conductor is small compared to the skin depth, the eddy current loss can be calculated with the 


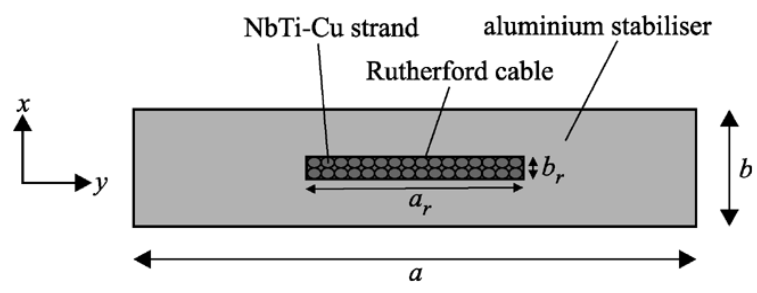

Fig. 2. Cross-section of conductor with strand, cable, and stabilizer. Definition of dimensions.

TABLE II

CONDUCTOR PARAMETERS

\begin{tabular}{ll}
\hline \hline Conductor type & Al stabilised NbTi-Cu cable \\
Conductor length & $6760 \mathrm{~m}$ \\
Dimensions of the conductor & $57 \mathrm{~mm} \times 12 \mathrm{~mm}$ \\
Dimensions Rutherford cable & $26 \mathrm{~mm} \times 2.3 \mathrm{~mm}$ \\
$\mathrm{No}^{\circ}$ of strands / diameter & 38 or $40 / 1.30 \mathrm{~mm}$ \\
$\mathrm{No}^{\circ}$ of filaments / diameter & $\approx 500 / \approx 40 \mu \mathrm{m}$ \\
filament twist pitch / transposition pitch & $50 \mathrm{~mm} / 180$ or $230 \mathrm{~mm}$ \\
$\mathrm{Al} \mathrm{RRR} / \mathrm{Cu} \mathrm{RRR}$ & $1200 \ldots 2400 / 180$ \\
Section of NbTi / Cu in the cable & $24.0 \mathrm{~mm}^{2} / 29.0 \mathrm{~mm}^{2}$ \\
\hline \hline
\end{tabular}

method that is described in [5]. Note that the magnet is ramped with a constant ramp rate and the formulas presented in [5] are for a sinusoidal varying field. The eddy current loss per unit of length $p_{\text {e.c. }}$, in $\mathrm{W} / \mathrm{m}$, for a rectangular conductor with dimensions $a \times b$ in a magnetic field with constant $\mathrm{d} B / \mathrm{d} t$ parallel to the $b$-side of the conductor (see Fig. 2) is:

$$
p_{\text {e.c. }}=\frac{a^{3} b}{12} \frac{1}{\rho}\left(\frac{\mathrm{d} B}{\mathrm{~d} t}\right)^{2},
$$

in $\mathrm{d} B / \mathrm{d} t$ is the rate of change of the magnetic field, $\rho$ the resistivity of aluminum. The resistivity of aluminum depends on the magnetic field, especially for fields up to $0.5 \mathrm{~T}$. This means that the eddy current loss power changes during a ramp. However, as will be explained in Section IV, the loss is measured at the end of the ramp where the change of the resistivity with the field is less strong. An average value is used in the calculation.

The ratio between the loss for field perpendicular and parallel to the wide face of the conductor is $(a / b)^{2}=23$.

\section{B. Filament Hysteresis Loss}

The filaments inside the strands are magnetized by the magnetic field. The twisted filaments inside the strands are fully decoupled with respect to the magnetic field at the low ramp rates that are used to power the magnet. Inside the windings the peak magnetic field $B$ during a ramp is much larger than the penetration field $B_{p}(<0.1 \mathrm{~T})$ of a filament. The mutual shielding of filaments can be neglected since the induced field is small compared to the applied field. Under these conditions, the power loss per unit length of conductor $p_{\text {hyst }}$, in W/m, is [6]:

$$
p_{\text {hyst }}=\frac{4 J_{c} d S_{N b T i}}{6 \pi} \frac{\mathrm{d} B}{\mathrm{~d} t}
$$

in which $d$ is the diameter of a filament, $S_{\mathrm{NbTi}}$ is the crosssectional area of $\mathrm{NbTi}$ in the cable, $\mathrm{dB} / \mathrm{dt}$ is the rate of change of the magnetic field and $J_{c}$ is the critical current density of the NbTi.

The critical current density of NbTi is a function of the magnetic field. This means that $J_{c}$ depends on the position in the windings and it also varies during a magnetic field cycle. For the calculation of the hysteresis loss in the magnet, an average value during the ramp time is used, depending on the position in the magnet.

Inside a magnet the filaments are exposed to magnetic field while simultaneously the transport current is changing. As shown in [7], the extra loss, compared to the hysteresis loss, can be taken into account by multiplying (2) with a factor $1+1 / 3 \cdot\left(I_{t} / I_{c}\right)^{2}$ in which $I_{t}$ is the amplitude of the transport current and $I_{c}$ the critical current. In the ATLAS Barrel Toroid magnets, which are operated well below their critical current, the correction is of the order of $1 \%$ and will be neglected. Note that the hysteresis loss is proportional to the ramp rate while the eddy current loss is proportional to the square of the ramp rate.

\section{Coupling Current Loss}

Currents are induced in the loops formed by the twisted filaments in the strands and the transposed strands in the Rutherford cable. These coupling currents have to pass normal conducting material to close the loop and thus generate ohmic losses. The time constants of these currents are very small [4] compared to the ramp time of the field because the contact resistances are relatively high. Therefore this type of loss is negligible.

\section{Coil Casing}

The double pancakes are embedded in an aluminum alloy (5083) casing, see Fig. 1. When the current in the coil changes, a current is induced in the casing which acts as a shorted secondary of a transformer with the coil as primary. The power $P_{\text {casing }}$, in $\mathrm{W}$, generated in the casing by a current with a ramp rate $\mathrm{d} I / \mathrm{d} t$, in the coil is:

$$
P_{\text {casing }}=\frac{U^{2}}{R_{\text {casing }}}=\frac{M^{2}}{R_{\text {casing }}}\left(\frac{\mathrm{d} I}{\mathrm{~d} t}\right)^{2},
$$

in which $U$ is the induced voltage, $R_{\text {casing }}$ is the resistance of the casing and $M$ is the mutual inductance between the coil windings and the casing.

There are also eddy currents induced in the coil casing by the changing magnetic field. However, due to the large resistivity of the casing material this loss component is negligible.

\section{E. Total Loss}

The total loss of the coil is the sum of (1), (2) and (3). For the calculation it is useful to rewrite the term $\mathrm{d} B / \mathrm{d} t$ in the conductor loss equations as $C \cdot \mathrm{d} I / \mathrm{d} t$, in which $C$ is the (local) coil constant in T/A and $\mathrm{d} I / \mathrm{d} t$ is the ramp rate in A/s. In Fig. 3 the total coil loss and the different loss components are plotted as a function of the ramp rate with a logarithmic $P$ axis to make the differences better visible. An 'average' $C$ of $5 \cdot 10^{-4} \mathrm{~T} / \mathrm{A}$ is used and an RRR of 1000 and 2500. The loss in the casing is by far the largest component. The eddy current loss in the stabilizer 


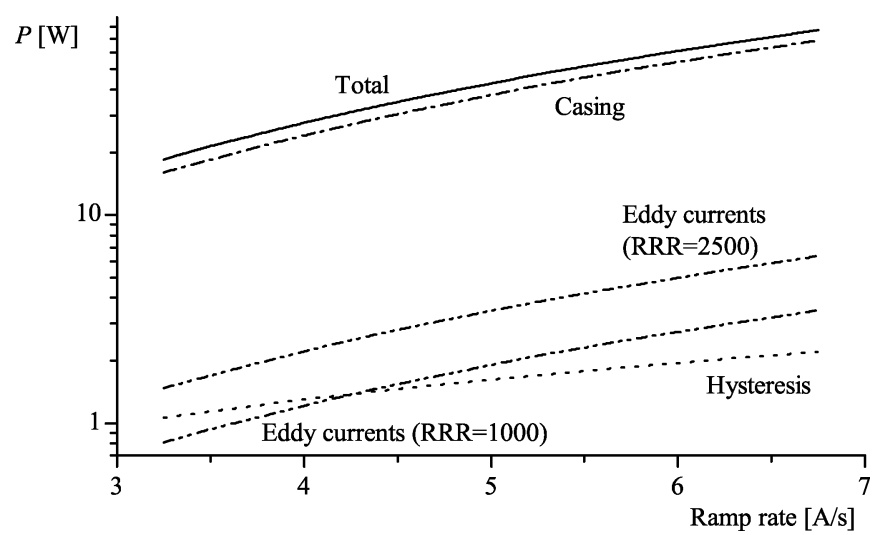

Fig. 3. Different loss components as function of ramp rate with $C=5$. $10^{-4} \mathrm{~T} / \mathrm{A}$ and $\mathrm{RRR}=1000,2500$

is the only term depending on the RRR. The difference for the two cases is almost a factor two in eddy current loss, but on the total it is very small and not visible on the scale used in Fig. 3.

\section{CoIl Loss CALCUlation}

An exact evaluation of the loss of the coil requires a 3D calculation of the magnetic field profile. Next, the AC loss of the conductor has to be calculated numerically, in 3D as well, because the magnetic field is not constant over the conductor volume. For this case no analytical formulas exist.

Since the conductor loss is only a small part of the coil loss, a simplified analytical approach can be used without too much loss of accuracy. The magnetic field is calculated in two dimensions in a cross-section of a coil which is considered to be infinitely long. The calculated magnetic field in the middle of the long sections of the racetrack is accurate in this approximation. Towards the corners the magnetic field is up to $15 \%$ underestimated.

The coil loss is calculated from the magnetic field in the middle of the cable with:

$$
P_{\text {coil }}=P_{\text {casing }}+P_{\text {conductor }}=P_{\text {casing }}+\sum_{i} L_{i} p_{i}\left(\mathbf{B}_{i}\right)
$$

in which $i$ is the winding number, $L_{i}$ is the length of winding $i$, and $p_{i}\left(\mathbf{B}_{i}\right)$ is the total AC loss per unit of length in winding $i$ with magnetic field $\mathbf{B}_{i}$.

Half of the cross-section that is used in the calculation of the magnetic field is shown in Fig. 4. The other half is identical but translated $4.5 \mathrm{~m}$ in the $-x$ direction. The individual cables in the pancakes are modeled as strips with a homogeneous current density, two for each of the four pancakes. The $B_{x}$ and $B_{y}$ component of the magnetic field are shown in Fig. 5 as a function of the position in the $x$ direction. The heights $y=53.9 \mathrm{~mm}$ and $114 \mathrm{~mm}$ correspond to the middle of the cable in the two upper pancakes, see Fig. 4.

The eddy current loss is mainly generated in the outer pancakes because there the $B_{x}$ component of the field is high compared to the inner pancakes. The hysteresis loss peaks increases towards the edges (in $x$-direction) of the pancakes because it depends on the total field.

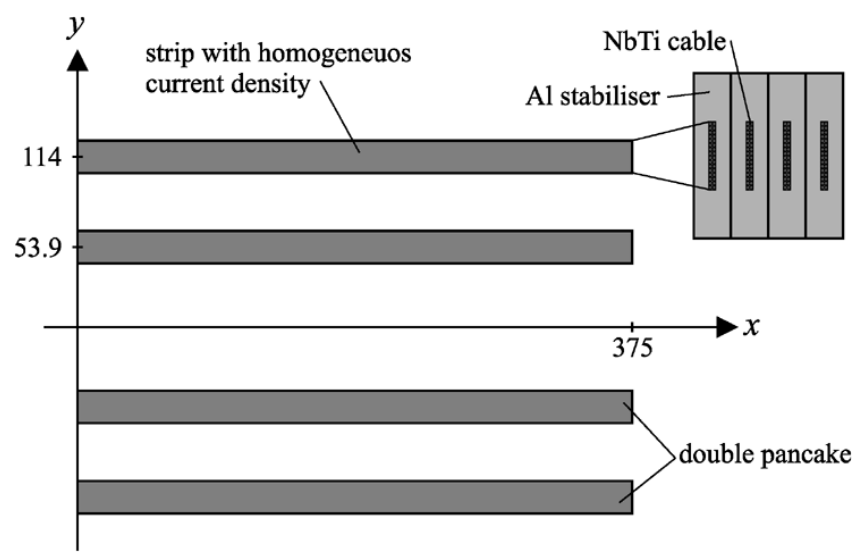

Fig. 4. Half of cross section used in 2-D calculation of magnetic field; four grey strips with homogeneous current density represent pancakes with NbTi cables in stabilizer (dimensions in millimeters).

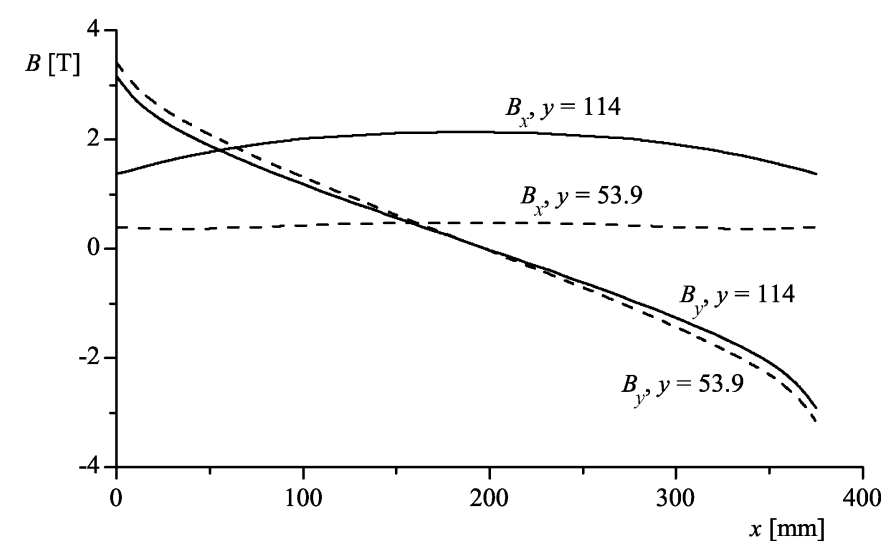

Fig. 5. Magnetic field $B_{x}$ and $B_{y}$ as function of position in $x$ direction in two upper pancakes for current of $22 \mathrm{kA}$. Heights $y=114 \mathrm{~mm}$ and $53.9 \mathrm{~mm}$ correspond to middle of cable in two upper pancakes (see Fig. 4).

Because of symmetry it is sufficient to calculate the loss in these two pancakes and double the result to obtain the loss for the complete coil.

\section{EXPERIMENT}

The coil is kept cold with two phase forced flow liquid helium flowing through cooling tubes that are attached to the coil casing. The helium is circulated with a centrifugal LHe pump immersed in a dewar which acts as a phase separator for the helium that returns from the coil [8]. The heat load is determined from the helium vapor mass flow from the phase separator to the refrigerator. The estimated uncertainty in the measurement is $15 \%$.

The ramp losses are measured at constant ramp rate as the difference between stable condition before the start of the ramp and stable condition during the ramp. It takes about 40 minutes after the start of the ramp to reach an equilibrium state.

What is measured in fact is an average of the loss power at the end of the ramp. So it is not the integrated loss of a complete field cycle which is the procedure usually followed for superconductors. The eddy current loss in the stabilizer (1) depends on the resistivity, which is a function of the applied magnetic field. This 
TABLE III

MEASURED RAMP LOSS AND CONDUCTOR RRR

\begin{tabular}{ccccc}
\hline \hline Coil No. & $4 \mathrm{~A} / \mathrm{s}$ & $5 \mathrm{~A} / \mathrm{s}$ & $6 \mathrm{~A} / \mathrm{s}$ & RRR [-] \\
\hline 2 & 26 & - & - & 1600 \\
3 & - & - & 44 & 1200 \\
4 & 30 & 49 & 67 & 2200 \\
5 & 31 & 46 & 68 & 1700 \\
6 & 28 & 48 & 67 & 1700 \\
8 & 25 & 47 & 62 & 2400 \\
\hline \hline
\end{tabular}

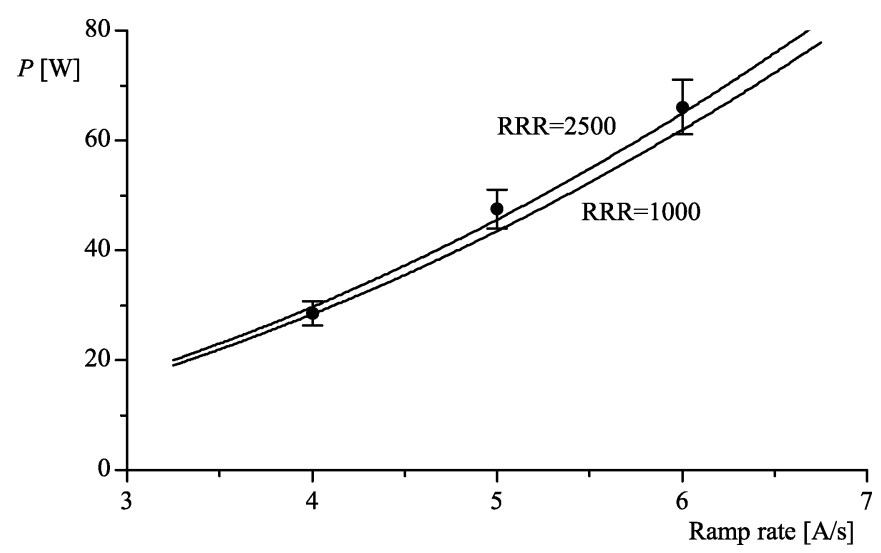

Fig. 6. Measured (symbols) and calculated (lines) ramp loss as function of ramp rate. Uncertainty in measured values is $15 \%$. Calculations are shown for two values of RRR.

loss contribution is thus not constant during the ramp. As discussed in Section II. A, an average can be taken without making a large error. The same holds for the critical current density $J_{c}$ in the hysteresis loss formula in (2).

\section{EXPERIMENTAL RESULTS}

During the test program of the eight Barrel Toroid coils, the ramp loss has been measured for 6 coils. The results are listed in Table III (- means: not measured). The last column of the table shows the measured RRR values of the various coils. The measurement of coil 3 is completely out of range and unfortunately only performed for one ramp rate so it is not possible to say whether this result is an error or not. Therefore this measurement is ignored. There are differences of a few watt between the various coils, but there is no relation with the measured RRR. The eddy current loss in the stabilizer is the only component that depends on the RRR, for the rest the loss should be identical for all coils. Apparently the few watt difference in eddy current loss is too small to be detected. Fluctuations between the different measurements, performed in a period of more than half a year, are too high.

For the comparison with the theoretically predicted loss, the average of the loss of coils 4, 5, 6 and 8 is plotted as a function of the ramp rate in Fig. 6 (symbols). The two lines represent the loss calculated with the method described in Section III. Two curves are shown, one with a high value of the RRR, one with a low value. Within the estimated uncertainty range of $15 \%$, the theoretically predicted loss is in excellent agreement with the experimental results.

\section{CONCLUSION}

During the on-surface test of the individual coils of the ATLAS Barrel Toroid, excellent agreement is found between the measured ramp loss and the theoretically predicted loss. The design capacity of the refrigerator for the ATLAS Barrel Toroid, which was based on the theoretical estimates, is thus sufficient to guarantee safe operation during ramp up and ramp down of the current.

The induced current in the coil casing, acting as a shorted secondary of a transformer, is responsible for 80 to $90 \%$ of the loss, depending on the ramp rate. The remaining loss is caused by hysteresis loss in the superconducting filaments and eddy current loss in the aluminum stabilizer of the cable. This last contribution is the only component that depends on the RRR of the conductor stabilizer. The uncertainty in the measurements was too large to see a relation between the RRR and the measured loss.

\section{REFERENCES}

[1] H. H. J. ten Kate, "ATLAS superconducting toroids and solenoid," IEEE Transactions on Applied Superconductivity, vol. 15, no. 2, pp. $1267-1270,2005$.

[2] A. Dudarev, J. J. Rabbers, S. Junker, R. Pengo, H. J. J. ten Kate, C. Berriaud, M. Arnaud, P. Vedrine, F. Broggi, and G. Volpini, "On-surface test of the ATLAS barrel toroid coils: Overview," presented at the 19th Int. Conf. Mag. Tech., Genova, September 18-23, 2005, unpublished.

[3] C. Berriaud, A. Dudarev, J. J. Rabbers, F. Broggi, S. Junker, L. Deront, S. Ravat, E. Adli, G. Olesen, R. Pengo, P. Vedrine, C. Mayri, E. Sbrissa, M. Arnaud, F. P. Juster, J.-M. Rey, G. Volpini, A. Foussat, P. Benoit, R. Leboeuf, M. Humeau, V. Stepanov, A. Olyunin, I. Shugaev, N. Kopeykin, and H. J. J. ten Kate, "On-surface test of the ATLAS barrel toroid coils: Acceptance criteria and results," presented at the 19th Int. Conf. Mag. Tech., Genova, September 18-23, 2005, unpublished.

[4] E. W. Boxman, A. Nijhuis, and H. H. J. ten Kate, "Alternating magnetic field losses in ATLAS type aluminium stabilized NbTi superconductors," Advances in Cryogenic Engineering, vol. 48, pp. 906-913, 2002.

[5] K. V. Namjoshi and P. P. Biringer, "Low-frequency eddy-current loss estimation in long conductors by using the moment of inertia of cross sections," IEEE Transaction on Magnetics, vol. 24, no. 5, pp. $2181-2185,1988$.

[6] N. Banno and N. Amemiya, "Analytical formulae of coupling loss and hysteresis loss in HTS tape," Cryogenics, vol. 39, pp. 99-106, 1999.

[7] W. J. Carr, "AC loss from the combined action of transport current and applied field," IEEE Transaction on Magnetics, vol. 15, no. 1, pp. 240-243, 1979.

[8] K. Barth, N. Delruelle, A. Dudarev, S. Junker, R. Pengo, O. Pirotte, and C. Berriaud, "Results of the cryogenic tests of the superconducting magnets forming the barrel toroid of the ATLAS experiment," presented at the CEC-ICMC '05, Keystone, Colorado, USA, 29/8-2/9 2005, unpublished. 